\title{
Vers un statut juridique du suisse-allemand ?
}

\author{
Bertil Cottier / Bastian Gossin ${ }^{*}$
}

Dans le prolongement de la controverse qui oppose l'usage du suisse-allemand au bon allemand dans des domaines aussi variés que l'éducation, l'immigration, la radiodiffusion, la défense nationale ou encore la procédure judiciaire, la présente contribution entend dresser l'état des lieux des dispositions juridiques, fédérales ou cantonales, qui régissent aujourd'hui le recours au dialecte. Au final, on en viendra à constater que le suisse-allemand est encore loin d'avoir acquis un réel statut juridique, sa forte présence à l'oral relève beaucoup plus de la tolérance que du droit. Que ce soit au niveau fédéral ou même au niveau cantonal, les textes légaux ne lui en effet accordent qu'une place marginale, sans proportion avec sa stature sociale.

I. Introduction 102

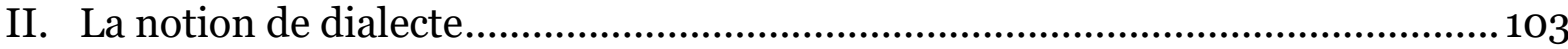

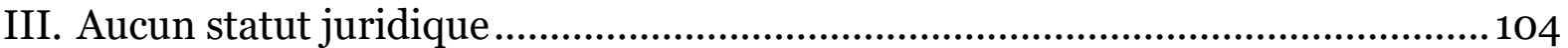

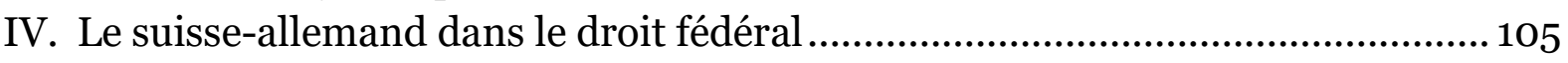

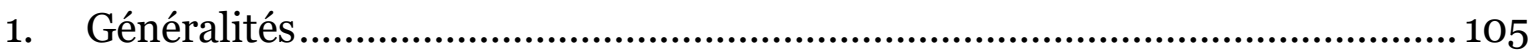

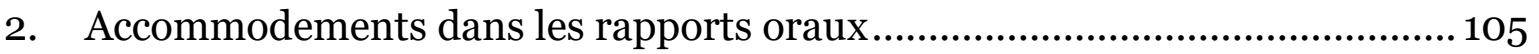

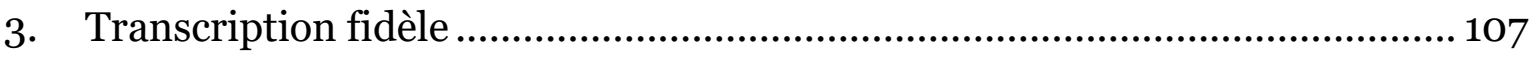

4. Intégration ou liens étroits avec la Suisse................................................. 108

V. Le suisse-allemand dans le droit cantonal .................................................... 109

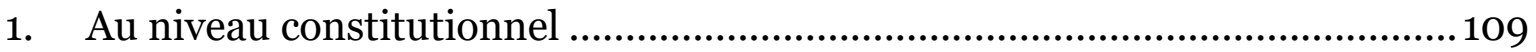

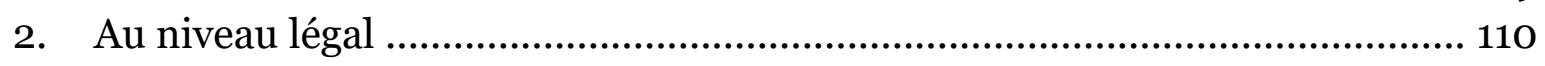

2.1. Les débats des organes législatifs..................................................... 110

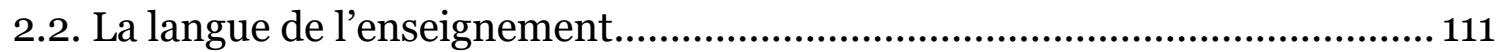

2.3. Le domaine de la culture......................................................................113

VI. Conclusion .........................................................................................114

Zitiervorschlag: Bertil Cottier / Bastian Gossin, Vers un statut juridique du suisse-allemand ?, in: sui-generis 2014, S. 101

URL: $\quad$ sui-generis.ch/9

DOI: $\quad$ https://doi.org/10.21257/sg.9

Bertil Cottier, professeur de droit de la communication (Université de la Suisse italienne et Université de Lausanne).

Bastian Gossin, master en Public Management and Policy (Université de Berne). 


\section{Introduction}

1 «Faut-il modifier l'article 4 de la Constitution et faire du suisse-allemand une langue nationale en lieu et place de l'allemand ? ». Dans la foulée de la décision du peuple argovien de bannir le «Hochdeutsch » des écoles enfantines, la question posée au Conseil fédéral, début juin de cette année, par le conseiller national tessinois Marco Romano avait assurément un côté provocateur ${ }^{1}$. Elle n'en reflétait pas moins une préoccupation: celle de voir le dialecte alémanique petit à petit supplanter le bon allemand sur la scène publique. Et menacer la cohésion nationale, l'allemand classique étant un vecteur d'échanges entre les communautés linguistiques du pays.

La réponse du Conseil fédéral s'est voulue rassurante. De la loi fédérale sur les langues aux directives sur la formation de la Conférence suisse des directeurs cantonaux de l'instruction publique, les moyens juridiques pour garantir un apprentissage du bon allemand ne manquent pas. D'où cette conclusion sèche et sonnante: «Il est hors de question d'amender la Constitution $»^{2}$.

3 La présente contribution n'entend pas prendre parti dans la controverse qui oppose le suisse-allemand au bon allemand. Son but est simplement d'alimenter objectivement le débat en faisant l'état des lieux des dispositions juridiques qui régis- sent aujourd'hui l'usage du suisse-allemand tant au niveau de la Confédération qu'au niveau des cantons; un tour d'horizon inédit, car jamais entrepris précédemment, à notre connaissance.

4 Faisant étape dans des domaines législatifs aussi variés que l'éducation, l'immigration, la radiodiffusion, la défense nationale ou encore la procédure judiciaire, notre inventaire du droit positif prendra en compte aussi bien les normes qui imposent le suisse-allemand que celles qui au contraire exigent une communication en «Hochdeutsch », ou, pour se conformer à la terminologie fédérale contemporaine, en allemand «standard». Les dispositions qui interdisent expressément l'usage d'une des deux langues sont elles aussi pertinentes pour notre recherche; toutefois, nous n'en avons recensé aucune. Ce qui nous amène à un premier constat : en matière linguistique, la technique législative est celle de la prescription, et non celle, plus discriminatoire, de la prohibition.

5 Enfin, il y a lieu de souligner que nous n'aborderons l'usage du suisse-allemand que dans le contexte public. Ailleurs prévaut la liberté des langues consacrée par l'article 17 de la Constitution. Cette disposition autorise l'usage de quelque idiome que ce soit dans les relations privées. C'est à ce titre que le suisse-allemand est pleinement protégé dans la vie familiale, associative ou commerciale3.

\footnotetext{
CN 14.5196. Le conseiller national Romano n'en est pas à son coup d'essai : deux ans auparavant, il avait déjà interpellé le Conseil fédéral sur l'opportunité d'enseigner le suisse-allemand dans les écoles des cantons latins ; il avait alors conclu son intervention en se demandant s'il ne fallait pas accorder au dialecte alémanique le statut de langue officielle au côté de l'allemand (CN
}

12.5384). Le conseiller fédéral Alain Berset avait répondu par la négative (Heure des questions, 3 décembre 2012).

2 Réponse du conseiller fédéral Berset (Heure des questions, 10 juin 2014).

3 Marco Borghi; La liberté de la langue et ses limites, in Daniel Thürer, Jean-François Aubert et Jörg Paul Müller (éd.), Droit constitutionnel 


\section{La notion de dialecte}

6 Pour les linguistes, le suisse-allemand ne constitue ni un dialecte, ni une langue en soi : il s'agit en fait d'un terme générique regroupant l'ensemble des nombreux dialectes alémaniques (lucernois, bâlois, haut-valaisan, etc.) parlés sur le territoire suisse4.

7 Reste à savoir ce que l'on entend par dialecte. De fait, distinguer un dialecte, par exemple l'alsacien, d'une langue, tel l'allemand «standard», n'est pas aisé ; en effet, tant un dialecte qu'une langue sont, tous deux, des systèmes de signes linguistiques destinés à communiquer. Les éléments de différenciation sont avant tout d'ordre sociolinguistique. Ainsi un dialecte a un ancrage régional : son usage est géographiquement restreint; de plus, il ne dispose en principe pas d'une normalisation, grammaticale et orthographique, officielle5.

Pareille difficulté de délimitation a conduit les auteurs de la Charte européenne des langues régionales ou minoritaires $\mathrm{du}$ 5 novembre $1991^{6}$ - un texte fondamental destiné à promouvoir les parlers en tant que composantes essentielles du patrimoine culturel de notre continent - à renoncer à donner une définition juridique de la notion de dialecte. Comme le souligne son rapport explicatif, la Charte « ne se prononce pas sur le point souvent controversé de savoir à partir de quand les différences d'expression sont telles qu'elles constituent des langues distinctes. Cette question dépend, non seulement de considérations proprement linguistiques, mais aussi de phénomènes psychosociologiques et politiques qui peuvent aboutir, dans chaque cas, à donner une réponse différente 7 ».

9 La Suisse, quant à elle, ne connaît pas de définition officielle de la notion de dialecte : aucun texte juridique, qu'il soit fédéral ou cantonal, ne précise le contenu de cette notion. La raison de ce mutisme tient certainement à la rareté de ce terme dans le vocabulaire juridique. Ce terme n'est utilisé que par quelques textes cantonaux (cf. infra 5) et par un seul texte fédéral: l'ordonnance du 4 juin sur les langues ${ }^{8}$; et encore, est-ce en rapport avec les aides financières à la presse romanche9. suisse, Zurich 2001, p. 608 N 8 et Regula KägiDiener, commentaire de l'art. 17 Cst., in Bernhard Ehrenzeller et alia (éd.), St. Galler Kommentar: Die schweizerische Bundesverfassung, 2ème édition, Zurich 2008, p. $408 \mathrm{~N} 24$.

4 Cf. Hadumod Bussmann (éd.), Lexikon der Sprachwissenschaft, 4ème édition, Stuttgart 2008, p. 610s.; Helen Christen, „Tour de Suisse“ der Deutschweizer Dialekte, in Forum Helveticum (éd.), Le dialecte en Suisse (alémanique) entre identité locale et cohésion nationale, Lenzburg 2005, p. 21 ; Beat Siebenhaar et Alfred Wyler, Dialekt und Hochsprache in der deutschsprachigen Schweiz, 5ème édition, Zurich 1997, p. 24ss. Walter Haas, Entre dialecte et langue l'exemple du Schwyzertütsch, in François Redart, René Jeanneret et Jean-Pierre Métral (éd.), Le
Schwyzertütsch 5e langue nationale ? Actes du colloque de la Commission interuniversitaire suisse de linguistique appliquée, Neuchâtel 1981, p. 22ss.

5 Pour plus de détails sur la différentiation entre langue et dialecte, cf. Hadumod Bussmann, op.cit. (note 4), p. 131s. ; Haas Walter, op.cit. (note 4), p. 22s.

6 La Charte a été ratifiée par la Suisse en 1997 (RS 0.441.2).

7 Rapport explicatif, ad 32.

8 OLang, RS 441.11.

9 Art. 21 OLang « Pour entrer en considération, une agence de presse doit notamment : $(.$.$) ; b. prendre$ en compte les dialectes romanches et le rumantsch grischun; ». 


\section{Aucun statut juridique}

10 On soulignera d'emblée que nulle part le suisse-allemand ne bénéficie d'une pleine reconnaissance juridique.

11 On sait que la constitution fédérale fait peu de cas du suisse-allemand : il n'est désigné ni comme langue officielle (art. 70), ni même comme langue nationale (art. 4) ; l'une et l'autre de ces dispositions font référence à l'allemand (sans autre précision).

12 On sait peut-être moins qu'aucune constitution cantonale ne mentionne le suisseallemand. Qui plus est, ce terme n'apparaît pas dans les deux seules législations sur les langues qui existent en Suisse : ni la loi fédérale sur les langues nationales et la compréhension entre les communautés linguistiques du 5 octobre 2007 (LLC) ${ }^{10}$, ni la loi sur les langues du canton des Grisons du 19 octobre 2006 n'en font état ${ }^{11}$.

Cela dit, il convient de se demander si le terme allemand, tel qu'utilisé par la Constitution fédérale, vise le seul allemand littéral ou s'il peut être interprété extensivement, et partant recouvrir aussi le suisseallemand. La réponse doit être nuancée selon que l'on envisage l'allemand en tant que langue officielle ou en tant que langue nationale.
14 De portée symbolique, le concept de langue nationale autorise une vision large des idiomes privilégiés ; large car elle renvoie aussi bien à la langue standard qu'à ses différentes variantes dialectales: patois ajoulot, patois tessinois, mais aussi Berndütsch, Züridütsch ou encore Walliserdütsch $^{12}$. Cette approche extensive s'inscrit parfaitement dans la logique du constituant qui entendait promouvoir l'identité nationale en accordant une valeur culturelle aux différents parlers de notre pays ${ }^{13}$. Ceux-ci sont considérés comme une composante essentielle de notre patrimoine ${ }^{14}$; partant, ils doivent non seulement être sauvegardés mais encore favorisés. En revanche, le concept de langue officielle est de nature exclusivement juridique : à la source de droits et d'obligations en ce qui concerne les rapports entre les autorités publiques et les citoyens ou entre elles, il doit être entendu restrictivement. C'est ainsi que l'article 5 alinéa 2 LLC précise que les organes fédéraux doivent utiliser «les langues officielles dans leur forme standard». En conséquence, seul l'allemand littéral bénéficie du statut de langue officielle.

15 Ce point de vue est partagé par la doctrine : aucun auteur ne s'est hasardé à élever le suisse-allemand au rang de langue officielle, par voie d'interprétation ${ }^{15}$. schen den schweizerischen Sprachgemeinschaften, ZB1 1981, p. 215. Voir également le Rapport du 15 septembre 2006 de la Commission de la science, de l'éducation et de la culture du Conseil national sur l'initiative parlementaire - Loi fédérale sur les langues nationales et la compréhension entre les communautés linguistiques, FF 20068513.

13 Marco Borghi (Les langues officielles et les langues nationales, in Daniel Thürer, Jean-François Aubert et Jörg Paul Müller, Droit constitutionnel suisse,

Zurich 2001, p. 593 N 2) considère les langues nationales comme le code identitaire de la collectivité sociale: «C'est par le biais de la notion de langue nationale qu'une partie essentielle de l'identité collective est greffée au pacte constituant. »

14 Ou encore «Kulturträger », selon l'expression de Regula Kägi-Diener (Sprachenordnung vor neuen Herausforderungen, AJP/PJA 1995, p. 450).

15 Voir notamment Alexandre Papaux, La langue de la procédure civile et pénale en droit suisse et comparé (Belgique, Espagne, Finlande et Canada), Etude de politique linguistique, de droit constitutionnel et de procédure, Bâle/Neuchâtel 2012, p. 231s. N 319. 
Nullement reconnu comme langue officielle au niveau national, le suisse-allemand, n'a rien à attendre du droit supérieur non plus. Le seul texte international qui traite directement du statut des langues, la fameuse Charte européenne des langues régionales ou minoritaires, exclut expressément de son champ d'application «les dialectes de la (des) langue(s) officielle(s) de l'Etat» (art. premier) ${ }^{16}$. Reste que cette disposition ne saurait être interprétée comme interdisant à la Suisse de reconnaître, un jour ou l'autre, le suisse-allemand comme langue officielle ${ }^{17}$.

\section{Le suisse-allemand dans le droit fé- déral}

\section{Généralités}

17 A dire vrai ce titre est trompeur, car à ce jour, le droit fédéral ne mentionne nulle part le suisse-allemand. Cela veut-il dire qu'il est pour autant ignoré ? Nullement. Toute obligation d'utiliser la langue standard, à l'instar de l'art. 5 al. 2 LLC dont il a déjà été question (cf. supra 3), n'est en fait rien d'autre qu'une interdiction indirecte d'utiliser le suisse-allemand... ${ }^{18}$.
18 Qui plus est, force est de constater que les obligations de s'exprimer en langue standard sont implémentées avec plus ou moins de rigueur : plutôt plus s'agissant de l'expression écrite, plutôt moins s'agissant de l'expression orale. Ainsi, des accommodements en faveur du suisse-allemand sont possibles non seulement lors d'entretiens ou de séances entre personnes capables de comprendre le dialecte, mais aussi s'il importe de ténoriser fidèlement les propos tenus ou encore de prouver son intégration sociale. Voyons cela de plus près.

\section{Accommodements dans les rap- ports oraux}

19 Qui a eu l'occasion de surprendre une réunion entre fonctionnaires fédéraux alémaniques, l'aura d'emblée remarqué : l'idiome entendu n'est pas l'allemand standard, mais le dialecte. Cette pratique, qui va pourtant contre le libellé de l'art. 5 al. 2 LLC, a trouvé sa consécration juridique dans les Instructions du 27 août 2014 du Conseil fédéral concernant la promotion du plurilinguisme ${ }^{19}$, une directive interne destinée à concrétiser les règles sur l'égalité des chances entre les employés des différentes communautés linguistiques posées par la LLC et
Seule voix dissidente, celle de Rudolf Viletta (op. cit., note 12 , p. 215) qui est d'avis que le suisse-allemand a acquis un statut semi-officiel à l'oral.

16 Voir aussi ATF 139 I 240 (considérant 6).

17 Le rapport explicatif à la Charte rappelle en effet, à son chiffre 32, qu'il appartient à chaque Etat partie de définir librement la ou les langue(s) officielle(s) en usage sur son territoire : « La charte ne concerne pas les variations locales ou les différents dialectes d'une même langue. (...). C'est donc au sein de chaque Etat, dans le cadre des processus démocratiques qui lui sont propres, qu'il reviendra aux autorités concernées de préciser à partir de quand une forme d'expression constitue une langue distincte. $\gg$.
18 Dagmar Richter (Sprachenordnung und Minderheitenschutz im schweizerischen Bundesstaat, Berlin 2005 , p. 28, 305s. et 390) parle dans ce cas de «Mundartverbot». Voir le Rapport du 15 septembre 2006 de la Commission de la science, de l'éducation et de la culture du Conseil national sur l'initiative parlementaire - Loi fédérale sur les langues nationales et la compréhension entre les communautés linguistiques, FF 2006 8532: «L'al. 2 règle l'usage des langues officielles par les autorités fédérales, excluant l'emploi des dialectes dans les entretiens formels. ».

19 FF 20146407 ss. 
l'OLang. Le chiffre 22 de ce texte prévoit que « En présence de personnes ne comprenant pas le dialecte, les employés s'expriment dans la langue officielle dans sa forme standard ». Interprétée a contrario, cette règle, qui n'est pas nouvelle ${ }^{20}$, autorise le suisse-allemand si aucun employé non alémanique ne participe à la réunion ou à l'entretien. Une solution pragmatique qui a l'approbation de la doctrine ${ }^{21}$.

Semblable réalisme guide l'usage du suisse-allemand au service militaire. Ainsi l'art. 57 du règlement de service de l'armée suisse ${ }^{22}$ stipule que le "supérieur s'exprime dans la mesure du possible dans la langue du subordonné. Dans les formations où l'on parle plusieurs langues, on emploie la langue littéraire. ». Plus concrètement: l'officier alémanique se fera comprendre de ses soldats francophones ou italophones dans leur langue maternelle, ou à défaut en haut allemand ${ }^{23}$.
21 Le droit judiciaire quant à lui impose l'usage d'une langue officielle pour la conduite de l'instance. Ainsi, outre-Sarine, l'allemand standard sera la langue du procès 24 : pas question, par exemple, de rédiger une demande ou une réplique en dialecte. Cette règle vaut en effet de manière absolue pour les actes et les écritures ${ }^{25}$. Son application est en revanche moins rigoureuse lors des débats; dans les tribunaux inférieurs, les plaideurs ou les témoins n'hésitent pas à parler dialecte pour autant que tous les protagonistes le comprennent ${ }^{26}$. Certains cantons ont d'ailleurs officiellement avalisé cette pratique libérale; c'est le cas notamment des Grisons: l'art. 7 al. 5 de la loi sur les langues permet, en accord avec les parties, d'utiliser une autre langue que les trois langues cantonales officielles, ce qui laisse la porte ouverte à l'usage du dialecte devant les tribunaux grisons. Même souplesse dans le canton de Fribourg : tout en soulignant quel'allemand écrit est la seule langue officielle judiciaire dans le district
20 Elle existait déjà dans les instructions précédentes datant de 2002 (voir le chiffre 5.2).

21 Voir en particulier Marco Borghi, op. cit. (note 13), p. 600 N 24, Regula Kägi-Diener, commentaire de l'art. 70 Cst., in Ehrenzeller Bernhard et alia (éd.), St. Galler Kommentar: Die schweizerische Bundesverfassung, 2ème édition, Zurich 2008, p. 1266 ; Marco Savoldelli, Die Amtssprachenregelung nach dem neuen Sprachengesetz des Bundes - Ihre Bedeutung für das öffentliche Prozessrecht, ZB1 2008, p. 478.

22 RS 510.107.0.

23 Autre exemple d'accommodement en faveur du dialecte : la Directive du 1er janvier 2010 de l'Office fédéral des transports sur les examens de capacité des conducteurs de véhicules moteurs des chemins de fer; son chiffre 1.8 prévoit que « Selon accord entre tous les participants, les examens peuvent être conduits en dialecte. »

24 Voir l'art. 129 du code de procédure civile, l'art. 67 al. 1 code de procédure pénale suisse, les art. 6 et 10 de la procédure pénale militaire, l'art. 54 de la loi sur le Tribunal fédéral, l'art. 3 de la loi sur l'organisation des autorités pénales de la Confédération et l'art. 36 de la loi sur le Tribunal fédéral des brevets.

25 Alexandre Papaux, op.cit. (note 15), N 433. Du même avis, Jean-Maurice Frésard, commentaire de l'art. 54, in Bernard Corboz et alia, Commentaire de la LTF, Berne 2009, p. 384 qui précise que « On évitera l'utilisation de mots ou d'expressions du dialecte ou d'origine locale ou encore d'anglicismes, sauf s'ils ont une incidence sur le litige $»$. Voir aussi l'art. 3 al. 4 de la loi sur les langues du canton des Grisons qui précise que les autorités cantonales emploient pour la correspondance écrite les langues officielles dans leur forme standard.

26 Marco Borghi, op.cit. (note 13), N 28; Nina Frei, commentaire de l'art. 129, in Heinz Hausheer et Hans Peter Walter (éd.), Berner Kommentar, Schweizerische Zivilprozessordnung, vol. I, Bern 2012, p. 1476; Adrian Staehelin, commentaire de l'art. 129, in Thomas Sutter-Somm, Franz Hasenböhler, Christoph Leuenberger (éd.), Kommentar zur Schweizerischen Zivilprozessordnung (ZPO), Zurich 2013 N. 7. 
alémanique de la Singine, la Cour de cassation pénale du Tribunal cantonal a donné son accord, en 1991, à l'usage du suisse-allemand, si l'une des parties ne comprend pas la langue standard ou si toutes les parties maitrisent le dialecte ${ }^{27}$.

Toujours dans le même esprit de tolérance linguistique, la loi fédérale sur la radio et la télévision (LRTV ${ }^{28}$ ) laisse la plus grande marge de manœuvre aux radiodiffuseurs alémaniques : libres à eux d'opter pour l'allemand standard ou le suisse-allemand dans leurs programmes29. Seule exception, mais de taille, l'obligation faite à notre diffuseur de service public, la SSR, de recourir, en règle générale, au haut allemand dans les émissions d'informations importantes, par quoi il faut entendre celles «susceptibles d'intéresser un public au-delà de la région linguistique et hors des frontières nationales » (art. 24 al. 5 LRTV) ${ }^{30}$. Pour l'Autorité indépendante d'examen des plaintes en matière de radio-télévision, le bulletin météorologique, quoiqu'entrant parfaitement dans cette catégorie, peut être présenté en dialecte à la télévision, car il est doublé de données écrites et/ou graphiques très complètes, lesquelles sont aisément compréhensibles pour des téléspectateurs ne parlant pas le suisse-allemand 31 .

23 Reste qu'il y a des situations où la solennité de l'événement commande l'usage de la langue officielle, gage de sérieux. Tel est le cas de la célébration du mariage civil qui ne peut pas se faire en dialecte, même si les parties le comprennent ${ }^{32}$.

\section{Transcription fidèle}

24 Les accommodements en faveur du suisse-allemand sont rarissimes dans l'expression écrite. C'est logique : aucun des dialectes alémanique n'est officiellement normalisé. On relèvera néanmoins deux exceptions, motivées par la nécessité de transcrire fidèlement les propos oraux.

25 La première découle l'art. 78 al. 2 du code de procédure pénale suisse, lequel dispose que « Le procès-verbal est rédigé dans la

fédérale sur la radio et la télévision, Berne 2014, p. 311.

31 «Praktisch alle wichtigen Informationen werden mit sachdienlichem und allgemein verständlichem Bildmaterial illustriert: Satellitenbilder; Anzeige von Temperatur, Luftdruck, Luftfeuchtigkeit, Windstärke und Windrichtung; die mit entsprechenden Symbolen (z.B. Sonne, Wolken, Niederschläge, Windrichtung), der Nullgradgrenze, der Maximalund Mindesttemperaturen für die verschiedenen Landesregionen auf einer Schweizer Karte dokumentierten Wetterprognosen für den kommenden Vormittag und Nachmittag sowie die ebenfalls mit entsprechenden Symbolen und Zahlen gekennzeichneten Wetterprognosen und Temperaturen in Europa wie auch für die kommenden fünf Tage in der Schweiz». Décision b. 527 du 30 juin 2006, considérant 4.6.

32 Toni Siegenthaler, Das Personenstandsregister Beurkundung, Verwaltung und Bekanntgabe der Personenstandsdaten, Zurich 2014, p. 336. 
langue de la procédure ; toutefois, dans la mesure du possible, les dépositions essentielles sont consignées dans la langue utilisée par la personne entendue. ». Cet alinéa a une conséquence importante sur le procès-verbal de débats qui se dérouleraient en dialecte: les déclarations des parties seront reformulées en langue standard33, à moins qu'elles ne portent sur des éléments décisifs pour l'issue du procès ; dans ce cas, on retranscrira au plus près les propos tenus 34 . Cela dit, la loi ne précise pas ce que l'on doit entendre par " dépositions essentielles », ce qui laisse une large marge d'appréciation à la personne qui tient le procès-verbal 35 . Marge relativement limitée dans le canton de Thurgovie, où l'ordonnance du Tribunal cantonal sur la procédure civile et pénale prévoit que les déclarations de personnes interrogées sont à reporter au procès-verbal, dans la mesure du possible mot pour mot et si nécessaire avec les expressions dialectales utilisées ${ }^{36}$.

La seconde exception se trouve à l'art. $4 \mathrm{al}$. 2 de l'ordonnance sur les noms géographiques37; cette disposition impose un libellé dans la langue écrite de la région linguistique concernée, à moins que cela ne soit pas possible ou pas judicieux. On notera que le canton de Bâle-Campagne prône une application plutôt libérale de cette règle : l'art. 3 de l'ordonnance sur les noms géographiques et les adresses de bâtiments impose l'emploi de la langue standard pour les noms de communes, de localités et les noms de rues. En revanche, les toponymes ( «Flurnamen») doivent être en règle générale écrits dans la langue parlée locale («in der ortsüblichen Sprechform $\left.\gg 3^{8}\right)$.

\section{Intégration ou liens étroits avec la Suisse}

27 La naturalisation facilitée de l'enfant étranger ou du conjoint de Suisse de l'étranger est accordée, notamment, à qui prouve "un lien étroit avec la Suisse » (art. 26, respectivement 28, de la loi fédérale sur l'acquisition et la perte de la nationalité suisse)39; dans ce sens, la connaissance du dialecte (à défaut d'une langue officielle) peut être un élément décisif40.

28 La maitrise de la langue locale est un facteur clef pour prouver son intégration sociale et partant obtenir la nationalité suisse. La question de savoir si la maîtrise du suisse-allemand suffit à justifier l'intégration est laissée aux cantons; les pratiques en la matière divergent. Ainsi l'ordonnance sur les droits de citoyenneté du canton de Schwyz prévoit que pour l'obtention de la naturalisation ordinaire, la preuve de la connaissance de la langue allemande peut se faire en dialecte ou en
33 Ce qui n'est pas toujours aisé ; il importe néanmoins que la personne concernée vérifie soigneusement la transcription pour déceler d'éventuelles inexactitudes (Robert Hauser, Erhard Schweri, Viktor Lieber, Kommentar zum zürcherischen Gesetz über die Gerichts- und Behördenorganisation im Zivil- und Strafprozess, Zurich 2012, p. 506).

34 Arrêt du Tribunal cantonal de Zurich du 3 juillet 2013.

35 Arrêt du Tribunal cantonal de Zurich du 11 novembre 2013.
36 Art. 69 al. 3 Verordnung des Obergerichts vom 27. Mai 2010 über die Zivil- und Strafrechtspflege.

37 RS 510.625.

38 Art. 3 al. 1 Verordnung vom 1. März 2011 über geografische Namen und Gebäudeadressen.

39 RS 141.0.

40 Philippe Meier et Hans Martin Stettler, Droit de la filiation, 4ème édition, Berne 2009 N 1463. Voir aussi l'arrêt du Tribunal administratif fédéral du 7 février 2013, C-1426/2012 (considérant 3.4). 
langue standard4․ De même à Uri, les candidats désirant obtenir le droit de citoyenneté communale et cantonale peuvent attester de leur savoir à choix en dialecte ou en allemand standard42. Dans le canton d'Argovie, les entretiens de naturalisation sont conduits en dialecte, sauf si le candidat requiert l'usage du bon allemand (« Hochdeutsch»)43. En revanche, l'ordonnance sur la citoyenneté du canton de Saint-Gall exige que le candidat comprenne la langue standard lorsqu'elle est parlée clairement 44 .

29 Un virage net en faveur de l'allemand écrit est amorcé par le projet de loi fédérale sur l'intégration des étrangers en discussion devant le Parlement fédéral ; le texte impose aux candidats à l'octroi ou à la prolongation d'une autorisation de séjour la connaissance de la langue nationale parlée au lieu de domicile (art. 58a nouveau), par quoi, il faut entendre, selon le Conseil fédéral, la langue standard (et non le dialecte)45.

\section{Le suisse-allemand dans le droit cantonal}

\section{Au niveau constitutionnel}

3 Surprise : la seule constitution cantonale à mentionner expressément le dialecte n'est pas suisse alémanique, mais romande. Dans la logique de la valeur identitaire et culturelle attachée aux parlers régionaux (cf. supra 3), l'art. 42 al. 2 de la Constitution jurassienne enjoint l'État et ses communes à veiller et à contribuer «à la conservation, à l'enrichissement et à la mise en valeur du patrimoine jurassien, notamment du patois. ». Plus surprenant encore, la plupart des constitutions alémaniques ne souffle mot de la langue nationale ou officielle du canton. Font exception les cantons d'Argovie, Bâle-Ville, Bâle-Campagne, Lucerne, Schwyz et $\mathrm{Zu}-$ rich dont les lois fondamentales consacrent «l'allemand» comme langue de communication avec les autorités publiques ${ }^{4}$.

31 Font exception aussi, les constitutions des cantons plurilingues, lesquelles désignent expressément les langues reconnues sur leurs territoires respectifs. Sans toutefois jamais cité le suisse-allemand, elles sont ouvertes à une conception large de la notion d'allemand (saut peut-être le canton de Fribourg).

32 A commencer par le canton de Berne où le dialecte jouit depuis longtemps d'une solide considération sociale 47 . Certes, l'art. 6 al. 1 de la constitution ne consacre expressément que le français et l'allemand en tant que «langues nationales et officielles du canton de Berne»48. Toutefois, les travaux préparatoires démontrent que le terme allemand englobe «jede Varietät
41 Art. 5 al. 2 Bürgerrechtsverordnung vom 5. Juni 2012.

42 Art. 7 al. 3 Verordnung vom 26. Oktober 2011 über die Eignungsvoraussetzungen für das Kantons- und Gemeindebürgerecht.

43 Art. 11 al. 2 Verordnung vom 25. September 2013 über das Kantons- und das Gemeindebürgerrecht.

44 Art. 2 al. 1 Verordnung vom 19. Oktober 2010 über das St.Galler Bürgerrecht ainsi que l'annexe à ce texte.

$45 \quad$ FF 20132161.
46 Art. 71a Cst. AG, art. 57 al. 1 Cst. BL, art. 76 al. 1 Cst. BS, art. 7 Cst. LU, art. 44 Cst. SZ et art. 48 Cst. $\mathrm{ZH}$.

47 Beat Siebenhaar et Alfred Wyler, op. cit. (note 4), p. 18.

48 La mention de langue nationale (en allemand Landessprache) est ici avant tout d'ordre traditionnel : «Eine Unterscheidung, wie sie die Bundesverfassung in Art. 4 BV 1999 (Landessprachen als Identitätsmerkmal des Staates) einerseits und Art. 70 BV 
der deutschen Sprache»49; autrement dit, tous les divers parlers bernois sont visés (et même plus : toutes les formes dialectales de l'allemand $\left.5^{\circ}\right)$.

33 Dans le même sens, l'article 3 alinéa 1 de la constitution du canton des Grisons considère que l'allemand, le romanche et l'italien sont des «Landesprachen ». En recourant à cette expression particulière, que l'on pourrait traduire par langue cantonale 51 , le constituant entendait reconnaître «sämtliche Ausdrucksdormen der jeweiligen Sprache, also neben der Hochsprache auch die Idiome und Dialekte $» 52$, ainsi qu'offrir une garantie du maintien («Bestandsgarantie») des différentes langues indigènes53. Enfin, l'art. 12 al. 1 de la constitution valaisanne de 1907 déclare la langue française et la langue allemande «nationales». Cette disposition «erweist sich schon durch seinen Wortlaut als eine relativ alte Norm, die noch keine Unterscheidung zwischen Landessprachen und Amtsprachen trifft, sondern diesen Komplex einheitlich regelt»54. Le terme de langue nationale englobe ainsi aussi bien la langue officielle que la langue nationale (ou cantonale).

\section{Au niveau légal}

34 On constatera d'emblée que les rapports entre dialecte et langue standard ont plus retenu l'attention des législateurs cantonaux que celle du législateur fédéral. Outre le principal point de friction qu'est la langue de l'éducation scolaire, la thématique est aussi abordée, même si les médias en ont moins parlé, dans d'autres secteurs importants tels la culture ou le fonctionnement des institutions publiques. On commencera par ce dernier domaine.

\subsection{Les débats des organes législatifs}

35 Quelques cantons permettent à leurs députés au parlement de s'exprimer non en allemand standard, mais en suisse-allemand. Le cas le plus remarquable est celui du canton de Berne où siègent pourtant plusieurs députés de langue française. L'article 76 alinéa 1 du règlement du 9 mai 1989 du Grand Conseil dispose en effet que: «Les députés et les membres du Conseil-exécutif s'expriment en allemand (dialecte ou allemand [sic]) ou en français»55. De même, l'article 144 alinéa 3 de la loi du 16 mars 1998 sur les communes souligne qu'au «sein des organes de la
1999 (Amtssprachen als technisch-funktionaler Begriff) andererseits kennt, ist im Kanton Bern nicht erforderlich, da es hier keine Kleinstsprache mit Sonderstatus gibt.», Dagmar Richter, op. cit. (note 20), p. 579.

49 Walter Kälin et Urs Bolz,. Handbuch des bernischen Verfassungsrechts 1995, Berne 1995, p. 246.

50 C'est la raison pour laquelle le terme trop restrictif de langue cantonale fut rejeté lors des travaux de révision de la constitution; voir sur ce point Iwar Werlen, Der zweisprachige Kanton Bern, Berne 2000, p. 171.

51 Les versions italienne et romanche parlent en effet de «lingue cantonali» respectivement de «linguas chantunalas». Cette différence terminologique s'explique sans doute par le fait que le terme de «Landessprache» n'a probablement pas d'équivalent direct dans les autres langues.
52 Botschaft der Regierung an den Grossen Rat, 10/2001-2002, p. 492; Christian Rathgeb, Kommentar zu Art. 3, in BMT Bänziger, Mengiardi, Toller \& Partner (éd.), Kommentar zur Verfassung des Kantons Graubünden, Coire 2006/2009, art. 3 N 15.

53 Botschaft der Regierung an den Grossen Rat, 2/2006-2007, p. 87.

54 Dagmar Richter, op. cit. (note 18), p. 801.

55 Une motion d'une députée du Jura Bernois (motion Amstutz, 038-2014) demandant que le suisse-allemand soit banni du Grand-Conseil a été rejetée, le 3 juin 2014, par 102 voix contre 40, après un débat hautement émotionnel; deux députés francophones du district Bienne ont même plaidé pour le rejet de la motion au motif que l'allemand standard est une langue étrangère. Ce rejet n'est pas le premier : par trois fois auparavant, le parlement cantonal avait voté en faveur du maintien du suisse-allemand. 
conférence régionale bilingue de Biel/Bienne-Seeland-Jura bernois, les débats ont lieu en allemand (ou en dialecte) et en français» 56 . Même solution à Glaris où l'ordonnance sur le parlement cantonal considère l'allemand (dialecte et langue écrite) comme langue des débats57. Enfin, dans le canton de Bâle-Ville, le dialecte est, avec la langue standard, considéré comme langue officielle du Conseil de bourgeoisie de la cité de Bâle58.

36 A ces cantons libéraux s'oppose le canton de Zurich dont le règlement du Grand Conseil contraint les députés à intervenir dans la « langue écrite »59. En Valais, une règle d'origine coutumière exclut aussi l'emploi du dialecte au parlement cantonal ${ }^{60}$.

\subsection{La langue de l'enseignement}

37 L'inventaire de la place du suisse-allemand dans le domaine éducatif offre une image tout sauf uniforme : certains cantons bannissent le dialecte de l'instruction publique, d'autres, de plus en plus nombreux, lui ouvrent les portes de certains établissements scolaires, mais le plus souvent il ne s'agit que des classes enfantines ou de certains cours particuliers du cycle primaire. Ainsi d'après une enquête me- née par la Conférence suisse des directeurs cantonaux de l'instruction publique, la langue standard était de mise durant l'année scolaire 2012-2013 dans tous les cantons au niveau secondaire 1 , dans les écoles de maturité gymnasiale (à l'exception de Nidwald, qui ne connait pas de règles cantonales à ce sujet) et dans les écoles de culture générale, avec quelques rares exceptions pour des branches accessoires telles que l'éducation physique ${ }^{61}$. Au degré primaire, les cantons d'Argovie, d'Appenzell Rhodes-Intérieures, d'Appenzell Rhodes-Extérieures, de Berne, des Grisons et d'Obwald autorisent l'usage du dialecte durant les premières années ou pour certains modules ${ }^{62}$. Les autres cantons alémaniques utilisent en principe uniquement la langue standard comme langue d'enseignement ${ }^{63}$.

38 Avant d'examiner plus en détail cette réalité contrastée, on rappellera une prémisse importante : aux termes de l'article 15 alinéa 1 LLC « les cantons veillent dans le cadre de leurs attributions à ce que la langue d'enseignement, en particulier sa forme standard, soit l'objet d'une attention particulière à tous les niveaux de l'enseignement. ». Traduction: la cohésion nationale impose une bonne connaissance
56 Règle reprise à l'art. 8. al. 1 de l'ordonnance du 24 octobre 2007 sur le règlement d'organisation des conférences régionales.

57 Art. 93 al. 1 Landratsverordnung vom 13. April 1994.

58 Art. 6 al. 1 Ausführungsbestimmungen vom 9. September 1986 zur Geschäftsordnung des Bürgergemeinderates der Stadt Basel.

59 Art. 13 al. 1 Geschäftsreglement des Kantonsrates vom 15. März 1999. Par ailleurs, le règlement zurichois sur le Synode de l'Eglise réformée dispose que les membres du Synode doivent utiliser la langue standard lors des débats, du moins en principe (ce qui n'exclut pas le suisse-allemand...); en revanche, les rapporteurs des bureaux, des fractions, des commissions ainsi que le conseil d'Eglise doivent s'exprimer exclusivement dans la langue standard (art. 43 Geschäftsordnung vom 15. März 2011 der Evangelisch-reformierten Kirchensynode).

60 Iwar Werlen, Verena Tunger et Ursula Frei, Der zweisprachige Kanton Wallis, Sierrre 2010, p. 94; cf. aussi Beat Siebenhaar et Alfred Wyler, op. cit. (note 4), p. 18s.

61 Cf. CDIP/IDES Enquête auprès des cantons, degré secondaire I, 2013, p.1s. ; CDIP/IDES Enquête auprès des cantons, école de maturité gymnasiale, 2013, p. 1 ; CDIP/IDES Enquête auprès des cantons, écoles de culture générale, 2013, p.1s.

62 CDIP/IDES Enquête auprès des cantons, degré primaire, 2013, p.1s.

63 ibidem. 
de la langue standard. Même souci du côté de la Conférence suisse des directeurs cantonaux de l'instruction publique, laquelle invite ses membres à développer « la langue locale officielle (langue standard) (...) de manière conséquente dès le début de la scolarité (degré préscolaire)»64. Elle ajoute qu'en « Suisse alémanique, vu l'usage du dialecte, la promotion de la langue standard s'avère particulièrement importante $» 65$. Quant au concordat HarmoS, il prévoit que les écoliers atteignent notamment «une solide culture linguistique dans la langue locale [dans la version allemande : «in der lokalen Standardsprache»] (maitrise orale et écrite) ${ }^{66 »}$.

Au niveau des textes juridiques, on retiendra que l'art 68a de la loi sur l'école du canton de Bâle-Ville détermine que tant le dialecte que la langue standard sont des objectifs éducatifs équivalents au jardin d'enfants ${ }^{67}$. L'ordonnance soleuroise sur les écoles de maturité institue la langue standard comme langue d'enseignement ${ }^{68}$. Dans ce même canton, l'ordonnance sur l'intégration des enfants et adolescents de langue étrangère met sur pied des cours en dialecte au jardin d'enfans, mais des cours en langue standard au niveau primaire ${ }^{69}$. Quant aux écoles professionnelles, elles sont tenues dispenser leurs cours en principe dans la langue écrite («Schriftdeutsch »)70.

40 À Zurich, la loi sur l'école publique prévoit que l'enseignement durant les deux premières années de scolarisation (jardin d'enfants) se fasse en principe en dialecte, puis à partir de la troisième année en principe en langue standard71. Le règlement pour les écoles de maturité cantonale pour adultes impose l'allemand standard en tant que langue d'enseignement ${ }^{22}$.

41 Dans le canton de Berne, une motion visant à faire du suisse-allemand la langue d'enseignement au jardin d'enfants n'a pas abouti devant le Grand Conseil73. La loi sur l'école obligatoire stipule que l'enseignement en langues porte notamment sur « une solide culture linguistique dans la langue locale (maîtrise orale et écrite) »74. L'ordonnance de direction sur la formation professionnelle, la formation continue et l'orientation professionnelle prévoit dans certaines situations un droit à des subventions pour des « cours de dialecte pour les francophones »75.

42 À Glaris, le règlement scolaire pour l'école de commerce impose aux enseignants l'emploi de la langue standard ${ }^{76}$. Une règle similaire se trouve dans le règlement sur l'école professionnelle cantonale de Ziegelbrücke77. Dans le canton de Schwyz, juin 2007 sur l'harmonisation de la scolarité obligatoire (concordat HarmoS).

67 Art. 68a Schulgesetz vom 4. April 1929.

68 Art. $8^{\text {bis }}$ al. 1 Verordnung vom 30. Juni 1997 über die gymnasialen Maturitätsschulen.

6969 Art. 6 et 9 Verordnung vom 7. Mai 1991 über die Integration fremdsprachiger Kinder und Jugendlicher.

70 Art. 1 Verfügung vom 14. Dezember 1989 über die
Festlegung der Unterrichtssprache an den Berufsschulen.

71 Art. 24 Volksschulgesetz vom 7. Februar 2005.

72 Art. 4 Schulordnung für die Kantonale Maturitätsschule für Erwachsene vom 4. Februar 1997.

73 Motion Hess du 25 novembre 2010, 238-2010.

74 «Eine umfassende Grundbildung in der lokalen Standardsprache (mündliche und schriftliche Sprachbeherrschung) » (art 10 al. 1 lit. a).

75 Art. 77 al. 1 lit. k.

76 Art. 11 al. 4 Schulordnung der Kaufmännischen Berufsfachschule Glarus vom 8. September 2008.

77 Art. 10 al. 5 Schulordnung der Berufsfachschule 
le règlement scolaire prévoit l'utilisation de la langue standard de façon séquentielle au jardin d'enfants et en principe permanente dès la 1re année primaire ${ }^{78}$. La loi sur l'école obligatoire du canton de Nidwald dispose que le bon allemand («Hochdeutsch») est en principe la langue d'enseignement79. L'usage de la langue standard au jardin d'enfants est l'affaire du plan d'études ${ }^{80}$. A Obwald, l'ordonnance sur la formation fixe la langue standard comme la langue à employer en principe à tous les niveaux scolaires ${ }^{81}$. Enfin, dans le canton d'Argovie, l'usage du dialecte est autorisé par certains plans d'études ${ }^{82}$.

43 Enfin, on mettra un terme à ce patchwork en relevant une singularité : l'université de Bâle conditionne l'admission aux études de logopédie à la maitrise d'un dialecte suisse alémanique ${ }^{83}$.

\subsection{Le domaine de la culture}

44 Dans ce domaine, on relèvera avant tout l'institution d'aides financières destinées à favoriser l'usage du dialecte. Ainsi à Glaris, la loi sur le soutien à la vie culturelle permet au canton de subventionner des activités contribuant à la mise en valeur («Pflege ») du dialecte ${ }^{84}$ (on songe notamment à la promotion du théâtre en parler local). Quant à la loi sur les langues du canton des Grisons, elle offre la possibilité aux autorités de soutenir des travaux scientifiques liés aux dialectes locaux $^{85}$. Enfin, dans le canton d'Obwald, le dialecte est considéré comme faisant partie des biens culturels dont le canton doit prendre soin ${ }^{86}$.

45 Tout laisse à croire que le but visé à chaque fois par le législateur de ces trois cantons périphériques n'est pas de favoriser le suisse-allemand en tant que tel - il n'en a guère besoin vu sa prédominance sociale -, mais de soutenir la variante dialectale locale; son authenticité serait en effet menacée par les parlers des grands centres urbains, voir même par l'usage toujours plus fréquent de termes de haut allemand au détriment des équivalents originaux.
Ziegelbrücke vom 24. September 2008.

78 Art. 9 Reglement vom 1. Februar 2006 über die Rechte und Pflichten der Lehrpersonen und Schülerinnen und Schüler an der Volksschule.

79 Art. 24 al. 1 Gesetz vom 17. April 2002 über die Volksschule.

8 Art. 24 al. 2 Gesetz vom 17. April 2002 über die Volksschule.

81 Art. 16 al. 1 Bildungsverordnung vom 16. März 2006.

82 Pour plus de détails voir les annexes respectivement de la Verordnung vom 19. Mai 2010 über die Mittelschulen (Mittelschulverordnung) et de la Verordnung vom 9. September 1991 über die Aargauische Maturitätsschule für Erwachsene.

83 Art. 3 al. 2 lit. a Studienreglement vom 27. April 2004 für Logopädie am Institut für Spezielle Pädagogik und Psychologie der Universität Basel (ISP). Une exigence similaire pour les logopèdes se trouve dans l'ordonnance sur le personnel dans le domaine hospitalier, cf. art. 44 al. 1 Verordnung vom 6. Dezember 2011 über die Fachpersonen und Betriebe im Gesundheitswesen. A l'inverse, on notera qu'à Bâle-Campagne, l'ordonnance sur les services de traduction requiert la maitrise orale et écrite du bon allemand (« hochdeutsche Sprache ») pour être admis au registre cantonal des traducteurs (art. 11 al. 1 lit. a Verordnung vom 7. Mai 2013 über das Übersetzungswesen); une obligation similaire est posée par l'ordonnance sur les traductions du canton de Zoug (art. 7 al. 1 lit. a Verordnung vom 12. November 2013 betreffend das Übersetzungswesen im behördlichen Verkehr) et par l'ordonnance concernant les interprètes du canton de Zurich (art. 10 al. 1 lit. a Dolmetscherverordnung vom 26./27. November 2003).

84 Art. 4 al. 1 lit. i Gesetz vom 7. Mai 1972 über die Förderung des kulturellen Lebens.

85 Art. 12 al. 1 lit. d Sprachengesetz des Kantons Graubünden vom 19. Oktober 2006.

86 Art. 5 al. 1 Verordnung vom 25. April 1985 über die Kulturförderung und Kulturpflege. 


\section{Conclusion}

46 Arrivé au terme ce tour d'horizon, on ne peut que constater que le suisse-allemand est encore loin d'avoir acquis un réel statut juridique. Que ce soit au niveau fédéral ou même au niveau cantonal, le droit ne lui accorde qu'une place marginale, sans commune mesure avec sa stature sociale.

47 En effet, si le dialecte est consacré par l'un ou l'autre texte de loi, c'est plutôt en raison de sa valeur culturelle; dans une rhétorique normative plus programmatique que juridique, le législateur émet le vœu que cette composante essentielle du patrimoine national soit conservée, voire promue. Cela dit, à l'exception notoire (et controversée) du domaine de l'enseignement du premier cycle dans certains cantons, le suisse-allemand n'est nulle part imposé dans le secteur public. Lorsque le droit impose, c'est alors pour prescrire l'allemand standard.

48 Avec succès à l'écrit où le haut allemand triomphe. Le dialecte ne le supplante qu'en cas de nécessité impérieuse de transcrire fidèlement une appellation géographique ou de ténoriser, mot à mot, une intervention ou une déposition.

49 A l'oral cependant, le suisse-allemand revient en force. Mais, sur la pointe des pieds et par la petite porte : mis à part une poignée d'habilitations légales (dont l'exemple le plus frappant demeure l'autorisation faite aux députés bernois et glaronnais de débattre en dialecte), son admission repose sur une pragmatique tolérance; que l'on peut trivialement résumer en une phrase : à quoi bon s'escrimer à parler une langue étrangère lorsque l'on est entre nous?

50 Tolérance donc, mais rien de plus. Même si tous les protagonistes d'une séance maitrisent le suisse-allemand à la perfection, rien n'empêche l'un d'eux d'exiger que la discussion se déroule en bon allemand, qui, fort de sa qualité langue officielle, a toujours le droit pour lui (au niveau fédéral en tout cas).

51 Qui plus est, la tolérance n'est pas sans limites: si l'un des protagonistes ne comprend pas le dialecte, l'allemand standard sera de mise (même dans les cantons alémaniques monolingues). Autre limite : la solennité de l'évènement, qui peut interdire l'usage du (trop informel) dialecte, comme c'est le cas pour la célébration du mariage civil, une prestation de serment ou encore la lecture du jugement. Dès lors, si statut juridique il y a, c'est tout un plus d'un statut d'accessoire qu'il s'agit !

52 Reste que tout peut soudainement basculer. Rien n'exclut qu'en butte à des sursauts identitaires plus ou moins contrôlés, la Suisse ne se réveille un jour avec une nouvelle langue officielle. Elle se retrouverait alors dans une situation linguistique identique à celle du Luxembourg. Ce petit pays accorde au luxembourgeois, qui pourtant lui aussi n'est guère plus qu'un parler germanique local ${ }^{87}$, le statut de troisième langue officielle, aux côtés du français et de l'allemand ${ }^{88}$. Et ce sans problème, car au Luxembourg, tout le monde parle luxembourgeois....
87 Il appartient cependant à la famille du moyen allemand.

88 Voir la loi du 15 février 1984 sur le régime des langues, et en particulier son article 3 qui dispose que : « En matière administrative, contentieuse ou non contentieuse, et en matière judiciaire, il peut être fait usage des langues française, allemande ou luxembourgeoise, sans préjudice des dispositions spéciales concernant certaines matières.». 